\title{
Antioxidative activity of statins and HDL-PON1 association in lacunar ischemic stroke with and without white matter hyperintensity
}

\author{
Hyun Goo Kang ${ }^{1}$, Jin Sung Cheong ${ }^{2}$, In Hwan Lim², Kyeong Ho Yun ${ }^{3}$, \\ Hyun Young Park ${ }^{2, *}$
}

\author{
${ }^{1}$ Department of Neurology, Research \\ Institute of Clinical Medicine of Jeonbuk \\ National University, Biomedical \\ Research Institute of Jeonbuk National \\ University Hospital, Jeonju, South Korea \\ ${ }^{2}$ Department of Neurology, Wonkwang \\ University School of Medicine, Regional \\ Cardiocerebrovascular Center, Iksan, \\ South Korea \\ ${ }^{3}$ Department of Cardiovascular \\ Medicine, Wonkwang University School \\ of Medicine, Regional \\ Cardiocerebrovascular Center, Iksan, \\ South Korea
}

\section{*Correspondence}

hypppark@wonkwang.ac.kr

(Hyun Young Park)

\begin{abstract}
Purpose: Statins (HMG-coA reductase inhibitors) protect vessels from atherosclerosis through various mechanisms, but the clinical significance of statin-induced high-density lipoprotein cholesterol (HDL) changes has not been established. We evaluated the effects of statin treatment on the antioxidative activities of HDLs in ischemic stroke patients with and without white matter hyperintensity (WMH).

Methods: From January to December in 2013, eighty-two ischemic stroke patients (57 men, 25 women; mean age $67.0 \pm 11.8$ years) at the Wonkwang Medical Center were recruited retrospectively and antioxidant activity was assessed via paraoxonase 1 (PON1) activity. We studied changes in the patients' lipid profiles and assessed PON1 activity in patients with and without WMH, at baseline and 8 weeks after treatment with rosuvastatin $10 \mathrm{mg} / \mathrm{d}$.

Results: All patients evaluated antioxidant activity using PON1 activity at admission. After 8 weeks of rosuvastatin treatment, the mean HDL concentration increased to 0.83 $\pm 10.1 \mathrm{mg} / \mathrm{dL}$. The HDL levels increased in 54 patients $(64.3 \%)$ and decreased in 30 patients $(35.7 \%)$. PON1 activity increased to $15.0 \%$ in all patients, regardLess of WMH after rosuvastatin treatment $(+25.4 \%$ in subjects without $\mathrm{WMH} ; P<0.001)$. Baseline PON1 activity modestly correlated with HDL levels $(\mathrm{r}=0.365, P=0.019)$; however, PON1 activity after treatment did not correlate with HDL levels $(\mathrm{r}=0.149, P=0.347)$. Conclusion: Our findings suggest that statins increase antioxidant activity, especially assessed via PON1 activity, in ischemic stroke patients who did not have WMH.
\end{abstract}

\section{Keywords}

Antioxidative effect; Ischemic stroke; Lipid profile; Paraoxonase 1; Statin; White matter hyperintensity

\section{Introduction}

Lowering cholesterol levels using statins has a protective effect on coronary and intracranial artery atherosclerosis [13]. Statins have an antioxidative effect by decreasing lowdensity lipoprotein (LDL) and increasing high-density lipoprotein (HDL) levels [3]. In general, statin treatment has been associated with a modest elevation of HDL levels [2]. The oxidation of LDLs leads to atherosclerotic lesions, and HDLs are known to have antioxidative properties that enable them to inhibit the oxidation of LDLs [3]. An enzyme in HDLs, HDLassociated enzyme paraoxonase 1 (PON1), plays an important role in these antioxidative processes [4]. PON1 is a calciumdependent esterase that hydrolyses aromatic carboxylic acid esters, organophosphates, and carbamates, and it is associated with HDL [5]. PON1 plays an important role in the general prevention of peroxidative damage to cell membranes and the oxidation of biomolecules, such as LDL [6]. PON1 activity has been found to reduce the incidence of coronary artery disease by preventing the progression of atherosclerosis [7]. However, its association with cerebrovascular diseases remains unknown.

Although the pathogenesis of white matter hyperintensity (WMH) is not clearly understood, it is considered a sign of small vessel disease (SVD) associated with atherosclerosis [2]. In the case of SVD, oxidative stress damages the vasculature, causes changes in blood flow and the bloodbrain barrier, and promotes neurodegenerative alterations in the brain tissue [8]. Oxidative stress is related to atherosclerosis, and atherosclerosis is a risk factor for stroke [8]. Therefore, oxidative stress may have an association with the risk of stroke [8]. And also, SVD is associated with oxidative stress. It is observed bilaterally and symmetrically on T2-weighted magnetic resonance imaging (MRI) [9]. In general, WMH is considered as a diffuse cerebral arteriolopathy, and it is related to endothelial dysfunction [10]. However, how patients 
with ischemic stroke are affected by the interactions between statin treatment-induced antioxidative activity and $\mathrm{WMH}$ is not clearly understood.

The antioxidative activities after statin therapy in patients with and without WMH were compared to define functional responses beyond HDL-C levels. Antioxidative activity in ischemic stroke patients was assessed by comparing lipid levels and PON1 activities at the beginning of and after 8 weeks of rosuvastatin treatment. We aimed to evaluate the antioxidative effects of statin treatment based on the presence of WMH. We tried to examine it using HDL-PON1, which is one of the antioxidative markers [8].

\section{Methods}

\subsection{Patients}

For this retrospective study, we evaluated 1,072 stroke patients (over 50 years old, 584 males and 488 females) who were hospitalized at the Wonkwang Medical Center (Iksan, South Korea) between January 2013 and December 2013. We excluded 332 pathents who had hemorrhagic stroke and 592 patients who had other mechanisms of ischmic stroke. Total 558 patients with large-artery atherosclerosis (232 patiens), cardioembolism (206 patients) and other mechanisms (120 patients), according to the Trial of Org 10172 in Acute Stroke Treatment (TOAST) classification [11], were excluded from the study. We also excluded 66 patients with a history of statin allergy, those receiving medication that could affect lipid levels (e.g., fish oil, niacin, ezetimibe, and probucol), and those (34 patients) with inflammatory, neoplastic, or infectious diseases. Only 82 patients with lacunar infarction, classified as small vessel occlusion according to the TOAST classification, were included (Fig. 1). All patients were treated in the same way according to the "Clinical Practice Guidelines for Stroke in Korea" [12]. All patients provided consent for the anonymization of the data in accordance with the protocol set forth by the review board of Wonkwang University Hospital (Iksan, South Korea, WKUHIRB-1460).

\subsection{Serum paraoxonase and arylesterase activities of PON1 enzyme}

Serum paraoxonase and arylesterase activities were determined using commercially available kits (Rel Assay Diagnostics, Mega Tip, Gaziantep, Turkey). Serum paraoxonase activity toward paraoxon was measured following the hydrolysis of paraoxon to p-nitrophenol and diethyl phosphate in the absence of $\mathrm{NaCl}$ (baseline activity). The molar extinction coefficient of p-nitrophenol was 17,000 $\mathrm{M}^{-1} \mathrm{~cm}^{-1}$ at a $\mathrm{pH}$ of 8 ; the results were expressed as $\mathrm{U} / \mathrm{L}$. Serum arylesterase activity was determined by the presence of phenol following the reaction of phenylacetate. The molar extinction coefficient of phenol was $4,000 \mathrm{M}^{-1} \mathrm{~cm}^{-1}$; the results were expressed as $\mathrm{kU} / \mathrm{L}$. The total antioxidative status (TAS) assay was calibrated with a stable antioxidant standard solution, which is traditionally called the Trolox equivalent, a vitamin $\mathrm{E}$ analog. The results were expressed as mmol Trolox Equiv./L.

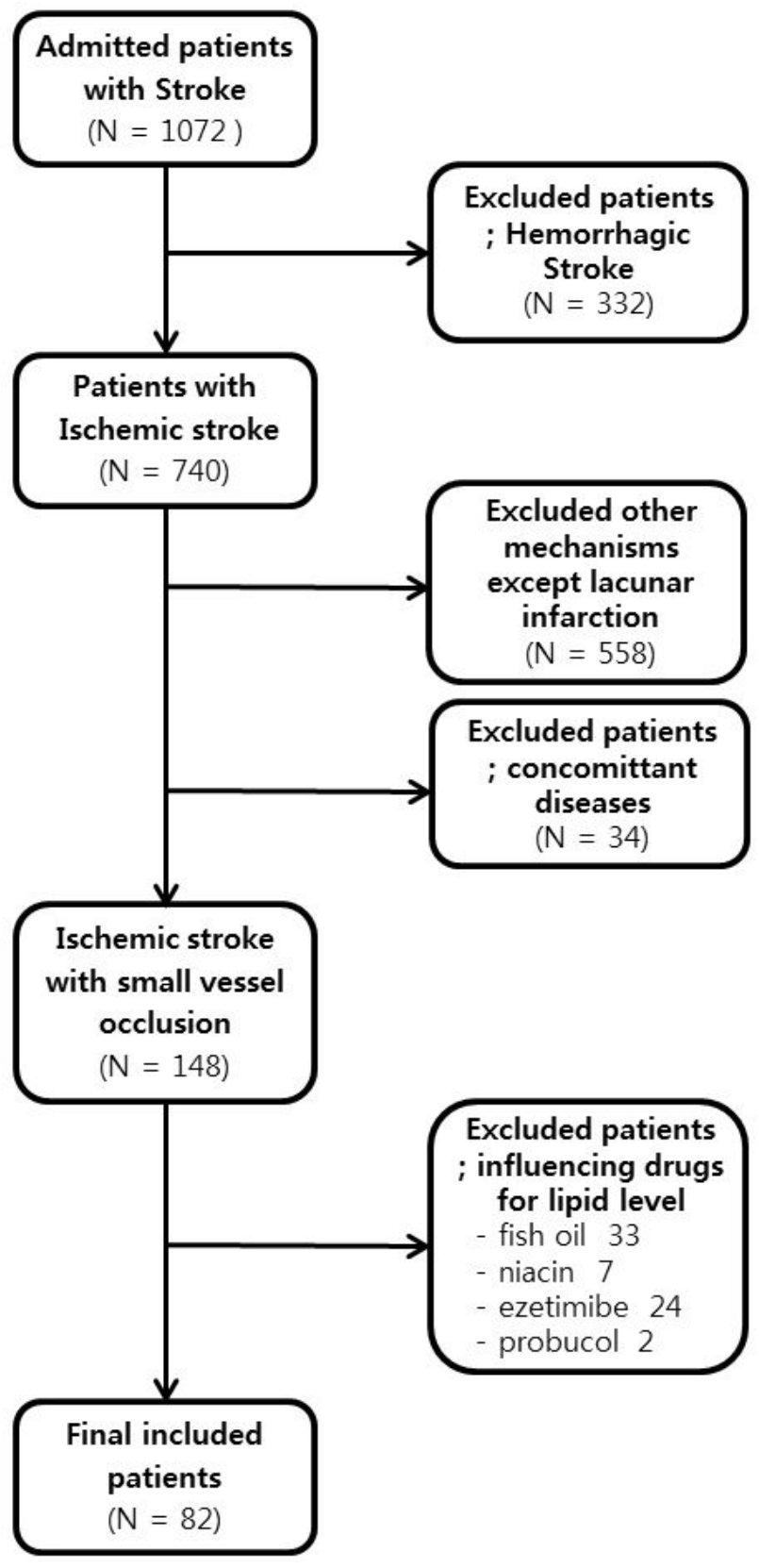

F I G U R E 1. Selection of ischemic stroke with small vessel occlusion in the study cohort.

\subsection{Data analysis}

We investigated the demographic characteristics (age and sex) and the medical history of all the patients (hypertension, diabetes mellitus, dyslipidemia, atrial fibrillation, coronary heart disease, previous stroke history, current smoking, and heavy alcohol drinking). Moreover, laboratory tests including lipid analyses were conducted. Rosuvastatin $10 \mathrm{mg} / \mathrm{d}$ (Crestor, Cambridge, United Kingdom) was administered to all the patients after they had enrolled for statin treatment. No patient withdrew from the study due to the adverse effects of statins. The treating physician prescribed anti-hypertensive drugs, such as angiotensin-converting enzyme inhibitors, betablockers, and nitrates.

Two neurologists (H.G.K and J.S.C) independently evaluated all brain MRI images of the patients. To prevent bias, these physicians were neither informed of the clinical condi- 
tions of the patients nor the laboratory assessments. Based on the Fazekas scale [13], WMH was assessed using white matter ratings. To evaluate and define the presence and absence of WMH, we classified Grade 0 on the scale as the absence of WMH and Grade 1 (or higher) as the presence of WMH.

Using an enzymatic colorimetric assay (U/L), total cholesterol $(\mathrm{mg} / \mathrm{dL})$, triglycerides $(\mathrm{mg} / \mathrm{dL})$, HDL-C $(\mathrm{mg} / \mathrm{dL})$, and LDL-C (mg/dL), in addition to lipid and lipoprotein $(\mathrm{mg} / \mathrm{dL})$ levels, were measured in patients who fasted overnight for 12 hours: first during the initial treatment phase (baseline) and after 8 weeks of treatment. The serum samples were analyzed using a fully automated paraoxonase activity measurement kit (Mega Tip, Gaziantep, Turkey) on the Hitachi 7600 automatic biochemical analyzer (Hitachi, Tokyo, Japan) to observe PON1 activity [14].

\subsection{Statistical analysis}

The demographic characteristics, medical history, and blood test results of the groups with and without WMH were compared. All the data were represented using either mean \pm standard deviation or percentage of patients (e.g., for categorical demographic characteristics). The categorical data were compared using the Chi-squared test, and Fisher's exact test was used when there were less than five variables. The continuous data were compared using independent two-sample $t$-tests. The Mann-Whitney $U$ test was employed for nonparametric data. Pearson correlation coefficients were calculated to quantify the relationships between changes in lipid profiles and white matter and PON1 activities. All the analyses were conducted using SPSS 21.0 (IBM Corp., Armonk, NY, USA), and statistical significance was set at $P<0.05$.

\section{Results}

A total of 1,072 stroke patients, $584(54.7 \%)$ males and 488 (45.3\%) females were hospitalized during the study period, and $740(69.1 \%)$ of them were diagnosed with ischemic stroke. Eighty-two patients, also diagnosed with small vessel occlusion, were finally selected for the study after excluding patients who satisfied any of the exclusion criteria listed above (see Methods section) (Fig. 1). The mean age of all the patients in the study was $67.0 \pm 11.8$ years, and 25 of them $(30.5 \%)$ were female. The patients were grouped into subjects with (36 patients; 43.9\%) and without (46 patients; 56.1\%) WMH based on the assessment of their MRI scans. The patients with WHM were significantly older than those without WHM, and the WMH group had a higher proportion of patients with diabetes than the non-WHM group (Table 1). In the group nonWMH group, no patient had previously suffered from cerebral infarction; twelve patients $(26.1 \%)$ had a history of cerebral infarction in the WHM group. No other obvious differences were found between the demographical data and laboratory findings related to the presence or absence of WMH (Table 1).

The changes in laboratory findings were evaluated after an 8-week statin treatment (Rosuvastatin $10 \mathrm{mg}$, Crestor, Cambridge, United Kingdom). RegardLess of the presence of WMH, total cholesterol $(\mathrm{mg} / \mathrm{dL}), \mathrm{LDL}(\mathrm{mg} / \mathrm{dL})$, and Apolipoprotein A1/B1 (mg/dL) levels significantly decreased after statin treatment in all patients (Table 2). However, for HDL (mg/dL) and PON1 (U/L) levels, we found different patterns. In the non-WMH group, both HDL $(+8.7 \%, P=$ $0.067)$ and PON1 levels $(+25.4 \%, P<0.001)$ increased. The group that showed white matter changes did not show any significant relationship between $\operatorname{HDL}(-2.6 \%, P=0.635)$ and PON1 $(+6.5 \%, P=0.123)$ levels over the course of the treatment (Table $2 \&$ Fig. 2). Furthermore, the baseline PON1 activities correlated with HDL-C levels $(\mathrm{r}=0.365, P$ $=0.019)$, unlike the PON1 activity after treatment, which did not correlate with HDL-C levels $(\mathrm{r}=0.149, P=0.347)$.

We also examined the TAS level before and after statin treatment. The pre-treatment TAS level in the non-WHM patients slightly increased after statin treatment (pre-treatment: 1.42 [1.39-1.56] mmol Trolox Equiv./I $\rightarrow$ post-treatment: 1.43 [1.39-1.46] mmol Trolox Equiv./I), but the difference was not significant. The pretreatment TAS level in the WHM patients slightly decreased after statin treatment (pre-treatment: 1.55 [1.39-1.69] mmol Trolox Equiv./I $\rightarrow$ post-treatment: 1.45 [1.42-1.55] mmol Trolox Equiv./I), but the difference was not significant.

\section{Discussion}

The objective of this study was to determine whether the antioxidative effects of statins were different in ischemic stroke patients with and without WHM. The main findings of this study are as follows. (1) Patients with WMH were significantly older, more often had diabetes, and were more likely to have a history of cerebral infarction than those without WMH. (2) Baseline laboratory findings showed that patients with WMH had significantly higher HDL and lower LDL and PON1 levels than patients without WMH. (3) After statin treatment, patients with WMH did not show any significant increase in HDL levels, but they showed a decrease in LDL levels. The PON1 antioxidative activity did not show any significant increase in patients with WMH. (4) In contrast, patients without WMH showed a significant decrease in LDL and increase in HDL levels and antioxidative activity after statin treatment.

Oxidative stress occurs when the physiological balance between oxidants and antioxidants is disrupted, causing damage to the organism [15]. Patients with early-stage ischemic stroke have low antioxidant levels [16]. Statins reduce cholesterol synthesis, and they affect oxidative stress through cholesterollowering independent effects (pleiotrophic effects). And also, there are beneficial effect when first-ever ischemic stroke occurs in patient who are already pretreatment with statin [17]. Reduced oxidation due to statins plays an important role in reducing the number of dead neurons in ischemic lesions or penumbra [18]. Owing to this effect, statin treatment improves post-stroke outcomes [19], and statin pretreatment reduces infarct volume in ischemic strokes [20]. PON1, assumed to have antioxidative activity, is an enzyme in HDLs. PON1 activity is therefore an indicator of anti-inflammatory and antioxidant activities of HDL [21]. Studies related to PON1 have been reported in many diseases such as multiple sclerosis associated with brain antioxidative effects [22] or psoriasis associated with cardiovascular disease [23]. However, an association between the decrease in PON1 activity observed 
T A B L E 1. Demographic characteristics, medical history, and laboratory findings of ischemic stroke patients with and without white matter hyperintensity (WMH).

\begin{tabular}{|c|c|c|c|}
\hline Variables & WMH $(-)(n=36)$ & WMH $(+)(n=46)$ & $P$-value \\
\hline \multicolumn{4}{|l|}{ Demographics } \\
\hline Age (years) & $60.89 \pm 11.91$ & $71.54 \pm 9.80$ & 0.003 \\
\hline Female & $10(27.8)$ & $15(32.6)$ & 0.742 \\
\hline \multicolumn{4}{|l|}{ Past History } \\
\hline Hypertension & $16(44.4)$ & $32(69.5)$ & 0.211 \\
\hline Diabetes mellitus & $4(11.1)$ & $30(65.2)$ & 0.001 \\
\hline Dyslipidemia & $6(16.7)$ & $4(8.7)$ & 0.636 \\
\hline Atrial fibrillation & $0(0.0)$ & $4(8.7)$ & 0.498 \\
\hline Coronary heart disease & $4(11.1)$ & $0(0.0)$ & 0.178 \\
\hline Previous stroke history & $0(0.0)$ & $12(26.1)$ & 0.029 \\
\hline Current smoking & $12(33.3)$ & $8(17.4)$ & 0.281 \\
\hline Heavy alcohol drinking & $6(16.7)$ & $12(26.1)$ & 0.708 \\
\hline \multicolumn{4}{|l|}{ Baseline laboratory findings } \\
\hline $\mathrm{HbA1c}(\%)$ & $6.08 \pm 0.96$ & $6.53 \pm 1.24$ & 0.213 \\
\hline hs-CRP (mg/dL) & $1.24 \pm 2.16$ & $1.56 \pm 2.62$ & 0.676 \\
\hline D-dimer (ng/mL) & $0.62 \pm 0.96$ & $0.54 \pm 0.49$ & 0.718 \\
\hline Fibrinogen $(\mathrm{mg} / \mathrm{dL})$ & $249.58 \pm 75.80$ & $283.65 \pm 79.88$ & 0.170 \\
\hline Fasting glucose (mg/dL) & $105.44 \pm 24.99$ & $125.71 \pm 73.94$ & 0.272 \\
\hline Total cholesterol (mg/dL) & $199.50 \pm 24.48$ & $191.75 \pm 16.09$ & 0.223 \\
\hline Triglyceride (mg/dL) & $143.22 \pm 56.06$ & $127.25 \pm 64.26$ & 0.405 \\
\hline HDL cholesterol (mg/dL) & $39.39 \pm 9.70$ & $45.17 \pm 9.59$ & 0.062 \\
\hline LDL cholesterol (mg/dL) & $118.67 \pm 21.34$ & $106.5 \pm 18.28$ & 0.054 \\
\hline Apoprotein A1/B1 (mg/dL) & $1.25 \pm 0.54$ & $1.38 \pm 0.38$ & 0.178 \\
\hline Total Antioxidant status (mmol Trolox Equiv./I) & $1.42[1.39-1.56]$ & $1.55[1.39-1.69]$ & 0.048 \\
\hline Paraoxonase $1(\mathrm{U} / \mathrm{L})$ & $258.89 \pm 105.43$ & $249.42 \pm 107.44$ & 0.604 \\
\hline
\end{tabular}

Values are presented as the percentage of patients (\%) or mean $\pm S D$ unless otherwise indicated. Antioxidant status is indicated by the median value.

$W M H=$ white matter hyperintensity; HbAlc $=$ hemoglobin Alc; hs-CRP $=$ high sensitive C-reactive protein; HDL cholesterol $=$ high-density lipoprotein cholesterol; $L D L$ cholesterol $=$ low-density lipoprotein cholesterol.

in ischemic stroke and $\mathrm{WMH}$ is yet to be established.

In general, WMH is a sign of small vessel diseases [24]. However, it can also be a symptom of aging, and it is frequently observed in patients with diabetes. When WMH is severe, it can influence cognitive function and cause vascular dementia. In the current study, patients with WMH were older, and a higher proportion had diabetes, which was consistent with the findings of previous studies [9, 24]. And silent multiple lacunar infarction such as WMH is associated with mild neuropsychological abnormalities in patients with firstever lacunar ischemic stroke [25]. The antioxidative effect of statins was not significant for patients with $\mathrm{WMH}$; there was a significant increase in antioxidative activity for patients without WMH. Endothelium-related vasodilatation dysfunction is induced by the loss of nitric oxide activity in vessel walls, and it disrupts cerebral vascular homeostasis [26]. In consequence, it can cause chronic hypoperfusion of white matter and trigger WMH. Reducing oxidative stress eventually improves endothelial function and decreases the risk of cardiovascular events [24]. In this study, statin treatment had a milder antioxidative effect, through PON1 activity, in patients with $\mathrm{WMH}$ than in those without WMH, suggesting that endothelial dysfunction influences the extent of the antioxidative effect.

\section{Limitations of the study}

This study has several limitations. First, to maintain the homogeneity of the sample, only a few patients were evaluated; they were patients with small vessel occlusion associated with WMH who visited a specific neurological center for ischemic stroke. Further analyses and large-scale studies are needed to confirm the results of this study. Second, this study was designed to evaluate the effects of rosuvastatin $10 \mathrm{mg}$, whereas other statins and their efficacies were not investigated. There- 


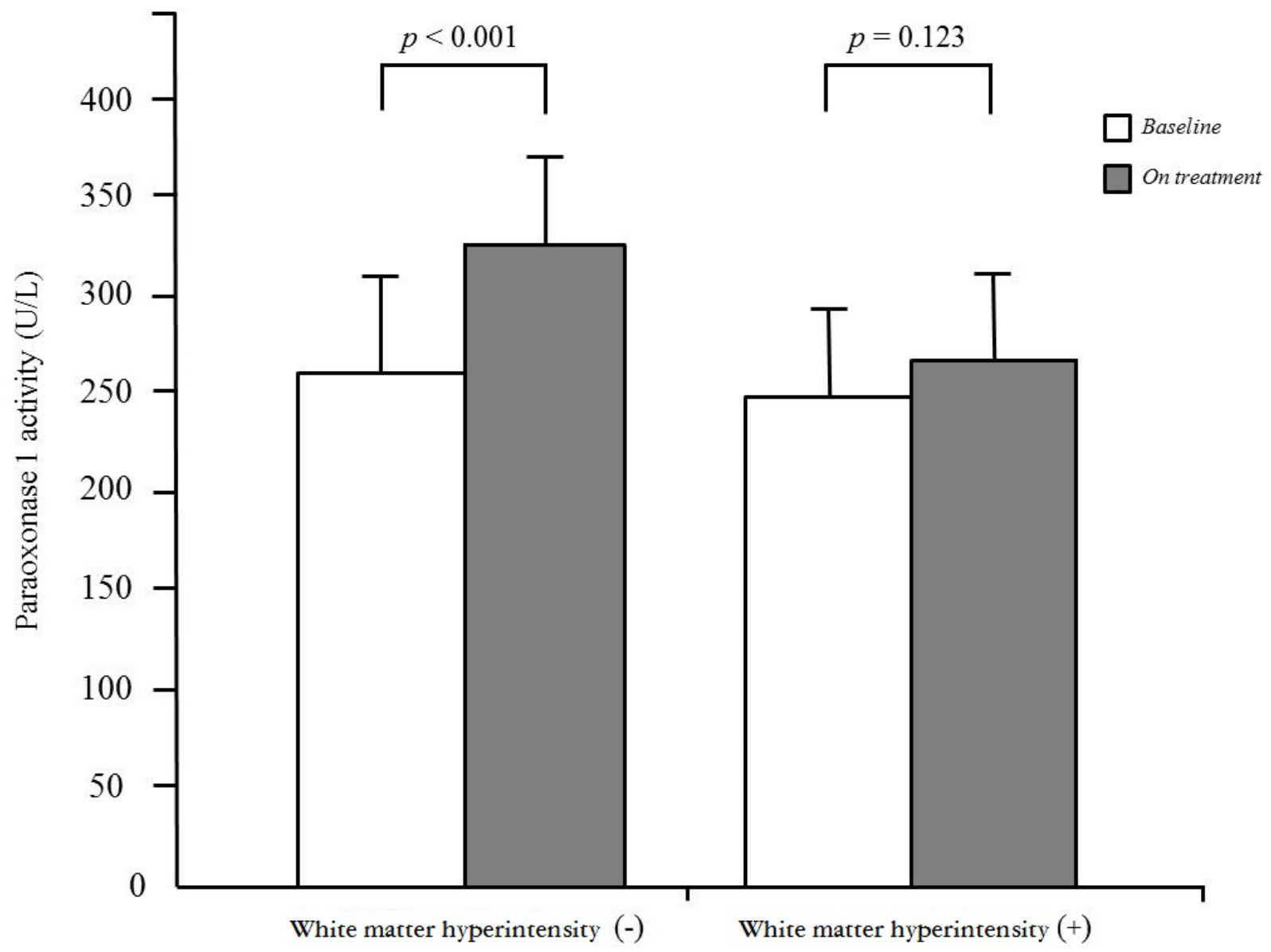

F I G URE 2. Changes in paraoxonase 1 activity after statin treatment stratified by white matter hyperintensity. To compare the change of paraoxonase 1 activity with or without white matter hyperintensity, we used paired $t$ test.

TA B L E 2. Comparison of laboratory findings before and after statin treatment.

\begin{tabular}{|c|c|c|c|c|}
\hline & Variable & Pre-treatment & Post-treatment & $P$-value \\
\hline \multirow{6}{*}{ WMH (-) } & Total cholesterol (mg/dL) & $199.50 \pm 24.48$ & $139.22 \pm 17.62$ & $<0.001$ \\
\hline & Triglyceride (mg/dL) & $143.22 \pm 56.06$ & $133.56 \pm 57.17$ & 0.543 \\
\hline & $\mathrm{HDL}(\mathrm{mg} / \mathrm{dL})$ & $39.39 \pm 9.70$ & $42.83 \pm 10.67$ & 0.067 \\
\hline & $\mathrm{LDL}(\mathrm{mg} / \mathrm{dL})$ & $118.67 \pm 21.34$ & $70.06 \pm 17.42$ & $<0.001$ \\
\hline & Apolipoprotein A1/B1 (mg/dL) & $1.12 \pm 0.12$ & $0.16 \pm 1.05$ & $<0.001$ \\
\hline & Paraoxonase 1 (U/L) & $258.89 \pm 105.43$ & $324.72 \pm 95.51$ & $<0.001$ \\
\hline \multirow{6}{*}{ WMH (+) } & Total cholesterol (mg/dL) & $191.75 \pm 16.09$ & $126.96 \pm 19.82$ & $<0.001$ \\
\hline & Triglyceride (mg/dL) & $127.25 \pm 64.26$ & $108.42 \pm 29.43$ & 0.088 \\
\hline & $\mathrm{HDL}(\mathrm{mg} / \mathrm{dL})$ & $45.17 \pm 9.59$ & $44.04 \pm 10.51$ & 0.635 \\
\hline & $\mathrm{LDL}(\mathrm{mg} / \mathrm{dL})$ & $106.50 \pm 18.28$ & $56.08 \pm 16.42$ & $<0.001$ \\
\hline & Apolipoprotein A1/B1 (mg/dL) & $1.31 \pm 1.34$ & $1.98 \pm 0.95$ & 0.034 \\
\hline & Paraoxonase 1 (U/L) & $249.42 \pm 107.44$ & $265.79 \pm 109.69$ & 0.123 \\
\hline
\end{tabular}

Values indicate mean \pm SD unless otherwise indicated.

$W M H=$ white matter hyperintensity; HDL cholesterol = high-density lipoprotein cholesterol; $L D L$ cholesterol $=$ low-density lipoprotein cholesterol.

fore, a large-scale study that explored varying types and doses of statins will be required in the future. Third, although we measured TAS within 24 hours after the onset of ischemic stroke, there was a slight difference in the sampling time for each patient. The slight difference in sampling time may have influenced TAS values, and the authors believe that it is a reason for the insignificance of the difference. However, unlike TAS, the PON values were significantly different. We speculated that TAS and PON responded differently to the slight sampling time difference. Therefore, we plan to con- 
duct another study with more patients. Last, the relationship between the antioxidative effects and the severity of WMH was not analyzed in this study. Future studies should analyze the different antioxidative effects related to $\mathrm{WMH}$ severity in a larger group of patients.

\section{Conclusions of the study}

In conclusion, we confirmed that statin treatment improved the lipid profiles of patients with ischemic stroke. However, statin treatment had a significantly greater antioxidative effect in ischemic stroke patients without WMH than in those with WMH. The results of the current study imply that the antioxidative effect of statin treatment is less in ischemic stroke patients with WMH than without WMH. Therefore, moderate- to highintensity statin treatments is thought to be helpful in secondary prevention of ischemic stroke in patients with $\mathrm{WMH}$.

\section{ETHICS APPROVAL AND CONSENT TO PARTICIPATE}

All patients provided consent for the anonymization of the data in accordance with the protocol set forth by the review board of Wonkwang University Hospital (WKUHIRB-1460).

\section{AUTHOR CONTRIBUTIONS}

Hyun Goo Kang and Hyun Young Park participated in the design of this research. Jin Sung Cheong, In Hwan Lim and Kyeong Ho Yun collected and analyzed the raw clinical data. Hyun Goo Kang, Kyeong Ho Yun and Hyun Young Park carried out computational studies and wrote the manuscript. All authors have read and approved the final manuscript.

\section{ACKNOWLEDGMENT}

I would like to express my gratitude to all those who helped me during the writing of this manuscript.

\section{FUNDING}

This study was supported by Wonkwang Grant in 2020 .

\section{CONFLICT OF INTEREST}

The authors declare no conflicts of interest.

\section{REFERENCES}

[1] Nissen SE, Tuzcu EM, Schoenhagen P, Brown BG, Ganz P, Vogel RA, et al. Effect of intensive compared with moderate lipid-lowering therapy on progression of coronary atherosclerosis: a randomized controlled trial. The Journal of American Medical Association. 2004; 291: 1071-1080.

[2] Tan TY, Kuo YL, Lin WC, Chen TY. Effect of lipid-lowering therapy on the progression of intracranial arterial stenosis. Journal of Neurology. 2009; 256: 187-193.

[3] Baigent C, Keech A, Kearney PM, Blackwell L, Buck G, Pollicino C, et al. Efficacy and safety of cholesterol-lowering treatment: prospective meta-analysis of data from 90,056 participants in 14 randomised trials of statins. The Lancet. 2005; 366: 1267-1278.
[4] Aviram M, Rosenblat M, Bisgaier CL, Newton RS, Primo-Parmo SL, La Du BN. Paraoxonase inhibits high-density lipoprotein oxidation and preserves its functions. a possible peroxidative role for paraoxonase. Journal of Clinical Investigation. 1998; 101: 1581-1590.

[5] Ferré N, Camps J, Fernández-Ballart J, Arija V, Murphy MM, Ceruelo $\mathrm{S}$, et al. Regulation of serum paraoxonase activity by genetic, nutritional, and lifestyle factors in the general population. Clinical Chemistry. 2003; 49: 1491-1497.

[6] Mackness M, Arrol S, Abbott C, Durrington P. Protection of low-density lipoprotein against oxidative modification by high-density lipoprotein associated paraoxonase. Atherosclerosis. 1993; 104: 129-135.

[7] McElveen J, Mackness MI, Colley CM, Peard T, Warner S, Walker CH. Distribution of paraoxon hydrolytic activity in the serum of patients after myocardial infarction. Clinical Chemistry. 1986; 32: 671-673.

[8] Brea D, Roquer J, Serena J, Segura T, Castillo J. Oxidative stress markers are associated to vascular recurrence in non-cardioembolic stroke patients non-treated with statins. BMC Neurology. 2012; 12: 65.

[9] WardLaw JM, Smith EE, Biessels GJ, Cordonnier C, Fazekas F, Frayne R, et al. Neuroimaging standards for research into small vessel disease and its contribution to ageing and neurodegeneration. The Lancet. Neurology. 2013; 12: 822-838.

[10] Lavallée PC, Labreuche J, Faille D, Huisse M, Nicaise-Roland P, Dehoux $\mathrm{M}$, et al. Circulating markers of endothelial dysfunction and platelet activation in patients with severe symptomatic cerebral small vessel disease. Cerebrovascular Diseases. 2013; 36: 131-138.

[11] Adams HP, Bendixen BH, Kappelle LJ, Biller J, Love BB, Gordon DL, et al. Classification of subtype of acute ischemic stroke. Definitions for use in a multicenter clinical trial. TOAST. Trial of Org 10172 in Acute Stroke Treatment. Stroke. 1993; 24: 35-41.

[12] Hong KS, Yu KH, Rha JH, Kim HY, Koo J, Park JM, et al. Review of evidences and updates of the Korean clinical practice guidelines for stroke in 2013. Journal of the Korean Neurological Association. 2013; 31: 143 157.

[13] Fazekas F, Chawluk J, Alavi A, Hurtig H, Zimmerman R. MR signal abnormalities at $1.5 \mathrm{~T}$ in Alzheimer's dementia and normal aging. American Journal of Roentgenology. 1987; 149: 351-356.

[14] Cebeci E, Oner FA, Usta M, Yurdakul S, Erguney M. Evaluation of oxidative stress, the activities of paraoxonase and arylesterase in patients with subclinic hypothyroidism. Acta Biomedica de l'Ateneo Parmense. 2011; 82: 214-222.

[15] Warlow C, SudLow C, Dennis M, WardLaw J, Sandercock P. Stroke. The Lancet. 2003; 362: 1211-1224.

[16] Cherubini A, Polidori MC, Bregnocchi M, Pezzuto S, Cecchetti R, Ingegni T, et al. Antioxidant profile and early outcome in stroke patients. Stroke. 2000; 31: 2295-2300.

[17] Arboix A, García-Eroles L, Oliveres M, Targa C, Balcells M, Massons J. Pretreatment with statins improves early outcome in patients with firstever ischaemic stroke: a pleiotropic effect of statins or a beneficial effect of hypercholesterolemia? BMC Neurology. 2010; 10: 47.

[18] Kishi T, Hirooka Y, Shimokawa H, Takeshita A, Sunagawa K. Atorvastatin reduces oxidative stress in the rostral ventrolateral medulla of stroke-prone spontaneously hypertensive rats. Clinical and Experimental Hypertension. 2008; 30: 3-11.

[19] Moonis M, Kane K, Schwiderski U, Sandage BW, Fisher M. HMGCoA reductase inhibitors improve acute ischemic stroke outcome. Stroke. 2005; 36: 1298-1300.

[20] Nicholas JS, Swearingen CJ, Thomas JC, Rumboldt Z, Tumminello P, Patel SJ. The effect of statin pretreatment on infarct volume in ischemic stroke. Neuroepidemiology. 2008; 31: 48-56.

[21] Mackness M, Mackness B. Paraoxonase 1 and atherosclerosis: is the gene or the protein more important? Free Radical Biology and Medicine. 2004; 37: 1317-1323.

[22] Salari N, Rasoulpoor S, Hosseinian-Far A, Razazian N, Mansouri K, Mohammadi $\mathrm{M}$, et al. Association between serum paraoxonase 1 activity and its polymorphisms with multiple sclerosis: a systematic review. Neurological Sciences. 2020.

[23] Oszukowska M, Kozłowska M, Kaszuba A. Paraoxonase-1 and other factors related to oxidative stress in psoriasis. Postepy Dermatologii I Alergologii. 2020; 37: 92-96. 
[24] de Leeuw FE, de Groot JC, Achten E, Oudkerk M, Ramos LM, Heijboer $\mathrm{R}$, et al. Prevalence of cerebral white matter lesions in elderly people: a population based magnetic resonance imaging study. The Rotterdam Scan Study. Journal of Neurology, Neurosurgery and Psychiatry. 2001; 70: 914.

[25] Blanco-Rojas L, Arboix A, Canovas D, Grau-Olivares M, Oliva Morera JC, Parra O. Cognitive profile in patients with a first-ever lacunar infarct with and without silent lacunes: a comparative study. BMC Neurology. 2013; 13: 203.

[26] Irace C, Cortese C, Fiaschi E, Scavelli F, Liberatoscioli L, Federici G, et al. The influence of PON1 192 polymorphism on endothelial function in diabetic subjects with or without hypertension. Hypertension Research. 2008; 31: 507-513.

How to cite this article: Hyun Goo Kang, Jin Sung Cheong, In Hwan Lim, Kyeong Ho Yun, Hyun Young Park. Antioxidative activity of statins and HDL-PON1 association in lacunar ischemic stroke with and without white matter hyperintensity. Signa Vitae. 2021;17(2):104-110. doi:10.22514/sv.2021.003. 